\title{
Health response in yellowtail Seriola dorsalis exposed to an Amyloodinium ocellatum outbreak
}

\section{Respuesta de salud en el jurel de Castilla (Seriola dorsalis) expuesto a un brote de Amyloodinium ocellatum}

\author{
Miroslava Vivanco-Aranda ${ }^{1}$, Oscar B Del Río-Zaragoza ${ }^{2 *}$, Claudia E Lechuga-Sandoval ${ }^{2}$, María \\ Teresa Viana ${ }^{2}$, Artur N Rombenso ${ }^{2}$ \\ ${ }^{1}$ Facultad de Ciencias Marinas, Universidad Autónoma de Baja California (UABC), Carretera Transpeninsular \\ Ensenada-Tijuana, no. 3917, CP 22860, Ensenada, Baja California, Mexico. \\ 2 Instituto de Investigaciones Oceanológicas, UABC. \\ * Corresponding author: E-mail: oscar.delrio@uabc.edu.mx
}

\begin{abstract}
Marine fish culture, both in hatchery and grow-out systems, is prone to parasitic infestations, which lead to fish health impairment and generally high mortality rates. The most frequent disease in these cultures, amyloodiniosis, is caused by the dinoflagellate Amyloodinium ocellatum, the parasite considered to inflict the most considerable damage on commercial marine fish ventures. In recent years, the yellowtail Seriola dorsalis cultured in Baja California has undergone recurrent parasitic infections. Thus, the objective of the present work was to evaluate the effects of a parasitic infection (A. ocellatum) in juvenile yellowtail in terms of mortality, gill histology, and blood parameters. Fish exposed to parasitic infection exhibited 100\% prevalence, with mean intensity of $766 \pm 500$ parasites per fish (grand mean \pm SD). Gill histological analyses indicated damage characterized by inflammation, epithelial detachment, hyperplasia, fusion of secondary lamellae, telangiectasia, and proliferation of mucous cells. Regarding blood parameters, red blood cell count, mean corpuscular hemoglobin concentration, and hemoglobin, glucose, and triglyceride concentrations were significantly higher $(P<0.05)$ in infected fish (parasite prevalence of $100 \%$ with a mean intensity of $882.19 \pm 265.05$ parasites per fish) than in healthy ones. Also, mean corpuscular volume, total protein, albumin, and globulin were significantly lower $(P<0.05)$ in infected fish than in healthy fish. No differences were found in the hematocrit, mean corpuscular hemoglobin, and white blood cell count $(P>0.05)$. This study demonstrated that $A$. ocellatum infection caused severe gill damage, affecting gas exchange efficiency, which resulted in blood parameter changes and, consequently, high mortality rates in a short-term period.
\end{abstract}

Key words: parasites, Seriola dorsalis, Amyloodinium ocellatum, blood parameters.

RESUMEN. El cultivo de peces marinos, tanto en el criadero como en el cultivo para engorda, es vulnerable a la incidencia de parásitos, lo que afecta la salud de los peces y, en general, conduce a altas tasas de mortalidad. La enfermedad más frecuente en estos cultivos, la amiloodiniosis, es causada por el dinoflagelado Amyloodinium ocellatum, considerado el parásito que inflige el daño más considerable a las empresas comerciales de peces marinos. En los últimos años, el jurel Seriola dorsalis cultivado en Baja California ha padecido infestaciones parasitarias recurrentes. Por lo tanto, el objetivo del presente trabajo fue evaluar los efectos de una infestación parasitaria (A. ocellatum) en juveniles de jurel en términos de mortalidad, histología de las branquias y parámetros sanguíneos. Los peces expuestos a la infestación parasitaria exhibieron una prevalencia del 100\% con una intensidad media de $766 \pm 500$ parásitos por pez (gran promedio \pm desviación estándar). El análisis histológico de las branquias indicó daño caracterizado por inflamación, desprendimiento epitelial, hiperplasia, fusión de las lamelas secundarias, telangiectasia y proliferación de células del mucus. Con respecto a los parámetros sanguíneos, el recuento de glóbulos rojos, la concentración de la hemoglobina corpuscular media, y las concentraciones de hemoglobina, glucosa y triglicéridos fueron significativamente mayores $(P<0.05)$ en peces infestados (prevalencia parasitaria del $100 \%$ con una intensidad media de $882.19 \pm 265.05$ parásitos por pez) que en peces sanos. Además, el volumen corpuscular medio, la proteína total, la albúmina y la globulina fueron significativamente más bajos $(P<0.05)$ en los peces infestados que en los peces sanos. No se encontraron diferencias en el hematocrito, la hemoglobina corpuscular media y los recuentos de glóbulos blancos $(P>0.05)$. Este estudio demostró que la infestación por A. ocellatum causó daños severos en las branquias que afectaron la eficiencia del intercambio de gases, lo que resultó en cambios en los parámetros sanguíneos y, en consecuencia, altas tasas de mortalidad en un corto periodo de tiempo.

Palabras clave: parásitos, Seriola dorsalis, Amyloodinium ocellatum, parámetros sanguíneos. 


\section{INTRODUCTION}

The yellowtail Seriola dorsalis (Gill 1863) is a coastal pelagic species found in subtropical and temperate regions. It is distributed along the Pacific coast of California (USA) and along the Baja California Peninsula and in the Gulf of California (Mexico) (Martinez-Takeshita et al. 2015). In Mexico, yellowtail catches in 2016 comprised nearly $455 \mathrm{t}$, with an approximate value of 611,000 USD (SEPESCABC 2017). Due to its high market value, adaptation to captivity (Poortenaar et al. 2001), rapid growth (Moran et al. 2009), and resistance to handling, this species is highly prized in recreational fisheries and is considered the target for aquaculture expansion in Mexico (commonly sold as hamachi or baja hiramasa in the seafood industry; Martinez-Takeshita et al. 2015). Currently, yellowtail commercial aquaculture operations are carried out mainly in the Baja California Peninsula, Mexico.

Marine fish culture, both in hatchery and grow-out systems, is prone to parasitic infestations, which lead to fish health impairment and generally to high mortality rates (Nakada 2002). The dinoflagellate Amyloodinium ocellatum causes one of the most serious diseases in fish species, amyloodiniosis, which inflicts the greatest damage on commercial fish aquaculture (Fajer-Avila et al. 2012). In the culture of the genus Seriola, the recurrent mortality outbreaks reported worldwide have been caused by ectoparasites, such as the protozoan $A$. ocellatum and the monogeneans Zeuxapta seriolae and Benedenia seriolae (Aiello and D'Alba 1986, Thoney and Hargis 1991, Montero et al. 2004, Reyes-Becerril et al. 2015).

The parasite A. ocellatum has a direct life cycle and it can easily multiply and disperse itself in confined areas, reaching very high infestation intensities that lead to mass mortality in fish cultures a few days from the outbreak. This parasite can also cause injuries in the gill tissue with several pathologies, such as lamellar fusion, high number of mucous cells, hyperplasia, and decreased gas exchange (Fajer-Avila et al. 2012), and clear changes in blood parameters. Monitoring fish health using blood analysis as a direct or inferential indicator is important and recommended. A blood analysis provides relevant information about the tolerance to a stressor agent and the health condition of fish under a parasitic outbreak (Del Rio-Zaragoza et al. 2008, Del Rio-Zaragoza et al. 2011).

In recent years, commercial aquaculture of yellowtail in Baja California has undergone numerous parasitic infection cases. Fortunately, no mass mortality events have been reported in this area (Reyes-Becerril et al. 2015). The present work thus aimed to evaluate the effects of parasitic infection (A. ocellatum) in juvenile yellowtail in terms of mortality, gill histology, and blood parameters.

\section{INTRODUCCIÓN}

El jurel Seriola dorsalis (Gill 1863) es una especie costera pelágica que se encuentra en regiones subtropicales y templadas. Se distribuye a lo largo de la costa del Pacífico frente a California, EUA, y a lo largo de la península de Baja California y en el golfo de California, México (Martinez-Takeshita et al. 2015). En México, las capturas de jurel en 2016 alcanzaron casi $455 \mathrm{t}$, con un valor aproximado de 611,000 dólares (SEPESCABC 2017). Debido a su alto valor comercial, adaptación al cautiverio (Poortenaar et al. 2001), rápido crecimiento (Moran et al. 2009) y resistencia a la manipulación, esta especie es altamente valorada en la pesquería recreativa y es considerada especie objetivo para la expansión de la acuicultura en México (comúnmente vendida como hamachi o baja hiramasa en la industria de alimentos marinos; Martinez-Takeshita et al. 2015). Actualmente, las operaciones de la acuicultura comercial se llevan a cabo, principalmente, en la península de Baja California, México.

El cultivo de peces marinos, tanto en el criadero como en el cultivo de engorda, es vulnerable a la incidencia de parásitos, lo que deteriora la salud del pez y, en general, conduce a altas tasas de mortalidad (Nakada 2002). Una de las enfermedades más serias en especies de peces es la amyloodiniosis, que es causada por el dinoflagelado Amyloodinium ocellatum e inflige el mayor daño en la acuicultura comercial de peces (Fajer-Avila et al. 2012). En el cultivo del género Seriola, los brotes recurrentes de mortalidad reportados mundialmente se deben a ectoparásitos, como el protozoo A. ocellatum y los monogeneos Zeuxapta seriolae y Benedenia seriolae (Aiello y Alba 1986, Thoney y Hargis 1991, Montero et al. 2004, Reyes-Becerril et al. 2015).

El parásito A. ocellatum tiene un ciclo de vida directo, y puede multiplicarse y dispersarse fácilmente en áreas confinadas y, así, alcanzar intensidades muy altas que llevan a la mortalidad masiva de peces en cultivo después de pocos días de iniciado un brote. Además, este parásito puede causar lesiones en el tejido de las branquias con varias patologías, como fusión lamelar, alto número de células mucosas, hiperplasia y disminución del intercambio gaseoso (Fajer-Avila et al. 2012), y cambios claros en los parámetros sanguíneos. El monitoreo de la salud de los peces mediante el uso de un análisis sanguíneo como un indicador directo o inferencial es importante y recomendable. El análisis sanguíneo provee información relevante sobre la tolerancia a agentes de estrés y la salud del pez durante un brote parasitario (Del Rio-Zaragoza et al. 2008, Del Rio-Zaragoza et al. 2011).

En años recientes, la acuicultura comercial del jurel en Baja California ha sufrido varios casos de infestación parasitaria. Afortunadamente, no se han reportado eventos de mortalidad masiva en el área (Reyes-Becerril et al. 2015). El presente trabajo tiene como objetivo evaluar los efectos de la infestación parasitaria (A. ocellatum) en juveniles de jurel en términos de mortalidad, histología de las branquias y parámetros sanguíneos. 
Vivanco-Aranda et al.: Health response in yellowtail exposed to an Amyloodinium ocellatum outbreak

\section{Material AND Methods}

\section{Fish and experimental system}

Juvenile S. dorsalis were obtained from Ocean Baja Labs (Eréndira, Baja California) through the Centro de Investigación Científica y de Educación Superior de Ensenada. Organisms $(n=530)$ were transported to our facilities at the Universidad Autónoma de Baja California (UABC). Upon arrival, fish $(n=30 ; 69.53 \pm 9.68 \mathrm{~g}$, grand mean $\pm \mathrm{SD})$ were stocked in a $10-\mathrm{m}^{3}$ tank with water exchange rate at approximately $10 \%$ per hour. Ten fish were examined and no signs of disease or parasites were observed. Fish were acclimated for 2 weeks in this tank. Two experiments were performed at different times to evaluate the effect of $A$. ocellatum on the gill tissue and examine blood parameter changes in infected fish.

In the 2 experiments, fish were randomly distributed in twelve 500-L tanks in a closed recirculating aquaculture system (RAS) connected to a PolyGeiser biofilter $\left(0.17 \mathrm{~m}^{3}\right)$. Filtered seawater passed through a $40-\mathrm{W}$ ultraviolet light. The RAS was equipped with an 1,100-L water reservoir and a 6,000-W titanium heater. Seawater was delivered to the tanks with a $1 / 3$ HP water pump, with daily water renewal set to $5 \%$.

A diet was formulated to contain $46 \%$ crude protein and $16 \%$ lipids as previously tested (Guerra-Olvera and Viana 2015, Rombenso et al. 2016). This diet was manufactured at the Laboratorio de Investigación y Desarrollo de Alimentos para Acuacultura (LINDEAACUA-UABC) under an extrusion process for floating feed $(3 \mathrm{~mm})$, in which an EXTRU-TECH E325 extruder was used and feed was dried in a horizontal air dryer (EXTRUTECH, USA). During both trials fish were hand fed 3 times a day (8:00, 13:00, and 18:00) until apparent satiation. Water temperature was maintained at $23.9 \pm 1.6{ }^{\circ} \mathrm{C}$, dissolved oxygen at $6.0 \pm 0.5 \mathrm{mg} \cdot \mathrm{L}^{-1}$, salinity at $34.6 \pm 0.4$, and $\mathrm{pH}$ at $8.1 \pm 0.3$.

\section{Parasitic induction}

In the first experiment, juvenile yellowtails $(n=96)$ were randomly distributed in 12 tanks ( 8 fish per tank) that had been previously exposed to a natural outbreak of A. ocellatum. Organisms were acclimated in the experimental system for $10 \mathrm{~d}$ at $18{ }^{\circ} \mathrm{C}$. Then, as a stress factor, water temperature was increased to $24 \pm 1{ }^{\circ} \mathrm{C}\left(1{ }^{\circ} \mathrm{C}\right.$ per day) to stimulate the development of the parasitic outbreak. Once water temperature reached $24{ }^{\circ} \mathrm{C}$, it was kept constant throughout the experiment. External signs of disease and fish mortality were monitored daily. Gills of dying fish were sampled at the end of the experiment.

\section{Gill analysis}

Gills from the right side of each dying fish were removed $(n=12)$ and placed on Petri dishes with sea

\section{MATERIALES Y MÉTODOS}

\section{Peces y sistema experimental}

Los juveniles de $S$. dorsalis fueron obtenidos de Ocean Baja Labs, a través del Centro de Investigación Científica y de Educación Superior de Ensenada. Los organismos $(n=530)$ fueron transportados a nuestras instalaciones en la Universidad Autónoma de Baja California (UABC). A su llegada, los peces $(n=30 ; 69.53 \pm 9.68 \mathrm{~g}$, gran promedio \pm desviación estándar) fueron sembrados en un tanque de $10 \mathrm{~m}^{3}$ con una tasa de recambio de agua de aproximadamente el $10 \%$ por hora. Diez peces fueron examinados, y no se encontraron signos de ninguna enfermedad o parásito. Los peces fueron aclimatados durante 2 semanas en este tanque. Se realizaron 2 experimentos a distintos tiempos para evaluar el efecto de A. ocellatum sobre el tejido branquial e investigar los cambios en los parámetros sanguíneos de los peces parasitados.

En los 2 experimentos, los peces fueron distribuidos al azar en 12 tanques de 500-L en un sistema de recirculación acuícola (RAS, por sus siglas en inglés) cerrado conectado a un biofiltro PolyGeiser $\left(0.17 \mathrm{~m}^{3}\right)$. El agua de mar filtrada pasó a través de luz ultravioleta de $40 \mathrm{~W}$. El RAS estaba equipado con un reservorio de agua de 1,100 L y un calentador de titanio de 6,000 W. El agua de mar fue distribuida a los tanques con una bomba de 1/3 HP y una tasa de renovación diaria del 5\%.

La dieta fue formulada para contener $46 \%$ de proteína cruda y $16 \%$ de lípidos con base en pruebas previas (Guerra-Olvera y Viana 2015, Rombenso et al. 2016). Esta dieta fue fabricada en el Laboratorio de Investigación y Desarrollo de Alimentos para Acuacultura (LINDEAACUA-UABC) bajo un proceso de extrusión para alimento flotante $(3 \mathrm{~mm})$, en el cual se utilizó un aparato de extrusión EXTRU-TECH E325 $y$ el alimento fue secado con un secador de aire horizontal (EXTRUTECH, EUA). Los peces fueron alimentados a mano 3 veces al día $(8: 00,13: 00$ y 18:00) ad libitum durante ambos ensayos. La temperatura del agua se mantuvo a $23.9 \pm 1.6^{\circ} \mathrm{C}$, el oxígeno disuelto a $6.0 \pm 0.5 \mathrm{mg} \cdot \mathrm{L}^{-1}$, la salinidad a $34.6 \pm 0.4$ y el $\mathrm{pH}$ a $8.1 \pm 0.3$.

\section{Inducción parasitaria}

En el primer experimento, los juveniles de jurel $(n=96)$ fueron repartidos al azar en los 12 tanques (8 peces por tanque) que previamente habían sido expuestos a un brote natural de $A$. ocellatum. Los organismos fueron aclimatados en el sistema experimental durante $10 \mathrm{~d}$ a $18^{\circ} \mathrm{C}$. Luego, como factor de estrés, la temperatura del agua fue incrementada a $24 \pm 1{ }^{\circ} \mathrm{C}$ ( $1{ }^{\circ} \mathrm{C}$ por día) para estimular el desarrollo del brote parasitario. Una vez que la temperatura del agua alcanzó $24{ }^{\circ} \mathrm{C}$, se mantuvo constante durante el resto del experimento. Los signos de enfermedad externos y la mortalidad de peces fueron monitoreados a diario. Las branquias de los peces moribundos fueron muestreados al final del experimento. 
water. Amyloodinium ocellatum was identified following the description by Lom and Dykova (1992). Parasites were counted using a stereomicroscope (Meiji, EMZ-TR; Tokyo, Japan) at $\geq 20 \times$. Prevalence (\%) and mean intensity of infection were determined as described by Bush et al. (1997).

Gills from the left side of each dying fish were removed and fixed in $10 \%$ buffered formalin. The second gill arch was then removed from the whole left gill, then dehydrated in graded ethanol series following standard histological procedures, and finally embedded in paraffin. Gills were stained with haematoxylin and eosin and mounted in acrylic resin of low viscosity (Cytoseal 60 Richard-Allan Scientific; Kalamazoo, MI, USA) for light microscopy.

\section{Blood parameters}

In the second experiment 132 juvenile yellowtails without parasites were randomly distributed in the 12-tank system (11 fish per tank), which was previously exposed to a natural outbreak of $A$. ocellatum. Fish were closely monitored, and blood samples were collected from dying fish. Fish from the same batch, but stocked in an independent RAS, were maintained under similar culture conditions to be used as control (healthy fish). No signs of disease or parasites were observed in this control group, and blood samples were also taken from this group.

\section{Blood sample collection}

Healthy fish $(n=23 ; 24.87 \pm 4.37 \mathrm{~g}, 12.97 \pm 0.88 \mathrm{~cm})$ and infected fish $(n=12 ; 38.41 \pm 10.62 \mathrm{~g}, 17.28 \pm$ $1.54 \mathrm{~cm}$ ) were carefully handled to minimize stress. Fish were anesthetized with $0.5 \mathrm{~mL} \cdot \mathrm{L}^{-1}$ of 2-phenoxyethanol (Sigma; St. Louis, MO, USA) following UABC protocols. In less than $3 \mathrm{~min}$, blood samples were collected from the caudal vein using $1 \mathrm{~mL}$ non-anticoagulant tuberculin syringes (BD PlastipakTM, Mexico). Blood aliquots were placed into 2 tubes immediately after sampling. The first tube had no anticoagulant and was used for the hematocrit test (HCT). The remaining blood was centrifuged for $10 \mathrm{~min}$ and the serum was stored at $-20{ }^{\circ} \mathrm{C}$ for analysis of total protein, albumin, globulin, glucose, and triglyceride concentrations. The second tube had K2EDTA (BD Microtainer; Franklin Lakes, NJ, USA) to prevent coagulation and was used for hemoglobin concentration, total red blood cell (RBC) count, and total white blood cell (WBC) count.

\section{Blood parameter analysis}

HCT was measured using a blood sample in a heparinized 2/3 filled capillary tube (LEEX Equipment, Mexico). The tube was sealed and placed in a micro-hematocrit centrifuge at 7,000 rpm (Premiere XC-3012, Mexico) for $10 \mathrm{~min}$.

\section{Análisis de las branquias}

Las branquias del lado derecho de cada pez moribundo fueron removidas $(n=12)$ y colocadas en cajas de Petri con agua de mar. Amyloodinium ocellatum fue identificado de acuerdo con la descripción de Lom y Dykova (1992). Los parásitos fueron contados con un estereomicroscopio (Meiji, EMZ-TR; Tokyo, Japón) a $\geq 20 \times$. La prevalencia (\%) y la intensidad media parasitaria fueron determinadas según lo definido por Bush et al. (1997).

Las branquias del lado izquierdo de cada pez moribundo fueron removidas y fijadas en $10 \%$ de formalina tamponada. Después, el segundo arco branquial fue removido de la branquia izquierda entera, deshidratado en una serie graduada de etanol de acuerdo con procedimientos histológicos estándar $\mathrm{y}$, finalmente, montado en parafina. Las branquias fueron teñidas con hematoxilina y eosina y embebidas en resina acrílica de baja viscosidad (Cytoseal 60 Richard-Allan Scientific; Kalamazoo, MI, EUA) para la microscopía de luz.

\section{Parámetros sanguíneos}

En el segundo experimento, 132 juveniles de jurel sin parásitos fueron distribuidos en el sistema de 12 tanques (11 peces por tanque), el cual había sido previamente expuesto a un brote natural de A. ocellatum. Los peces fueron monitoreados de cerca, y las muestras de sangre fueron tomadas de los peces moribundos. Los peces del mismo lote, pero colocados en un RAS independiente, fueron mantenidos bajo condiciones de cultivo similares para su uso como control (peces sanos). No se observaron signos de enfermedad o parásitos en el grupo control, y también se tomaron muestras de sangre de este grupo.

\section{Recolección de las muestras de sangre}

Los peces sanos $(n=23 ; 24.87 \pm 4.37 \mathrm{~g}, 12.97 \pm 0.88 \mathrm{~cm})$ y los peces parasitados $(n=12 ; 38.41 \pm 10.62 \mathrm{~g}, 17.28 \pm$ $1.54 \mathrm{~cm}$ ) fueron manipulados cuidadosamente para minimizar el estrés. Los peces fueron anestesiados con $0.5 \mathrm{~mL} \cdot \mathrm{L}^{-1}$ de 2-fenoxietanol (Sigma; St. Louis, MO, EUA) de acuerdo con los protocolos de UABC. En menos de 3 min, se tomaron las muestras de sangre de la vena caudal con jeringas de $1 \mathrm{~mL}$ de tuberculina sin coagulante (BD PlastipakTM, Mexico). Inmediatamente después del muestreo, las alícuotas de sangre fueron colocadas en 2 tubos. El primer tubo sin anticoagulante fue utilizado para la prueba del hematocrito (HCT). El resto de la sangre fue centrifugada por $10 \mathrm{~min}$ y el suero fue almacenado a $-20^{\circ} \mathrm{C}$ para el análisis de las concentraciones de proteínas totales, albúmina, globulina, glucosa y triglicéridos. El segundo tubo contenía K2EDTA (BD Microtainer; Franklin lakes, NJ, EUA) para evitar la coagulación y fue usado para la concentración de hemoglobina, el recuento total de glóbulos rojos (GR) y el recuento total de glóbulos blancos (GB). 
The packed cell volume was measured using a hematocrit reader and reported as a percentage (Del Rio-Zaragoza et al. 2008). Hemoglobin in erythrocytes was determined using a HemoCue $\mathrm{Hb} 201$ analyzer following the manufacturer's instructions (HemoCue AB; Angelholm, Sweden). The Natt and Herrick's (1952) method was used for total RBC and WBC-plus-thrombocyte counts. Cells were counted using a Neubauer hemacytometer under an optical microscope (Zeiss, PrimoStar; Göttingen, Germany). Mean corpuscular volume, mean corpuscular hemoglobin $(\mathrm{MCH})$, and mean corpuscular hemoglobin concentration (MCHC) were calculated by using standard formulas with the HCT, RBC, and hemoglobin data. Total protein, albumin, globulin, glucose, and triglycerides were determined from blood serum using colorimetric kit assays (MexLab Group; Jalisco, Mexico) following the manufacturer's instructions. Globulin was obtained by subtracting the value of albumin from total protein.

\section{Statistical analyses}

All measurements were done in triplicate for each fish. The normality test and $t$-test $(P<0.05)$ were applied to all data using SigmaStat 4 software (Systat Software; San Jose, CA, USA). All data reported in percentages were arcsine transformed before statistical comparisons. A non-parametric test (Mann-Whitney rank sum test) was used for data that failed to show normality.

\section{Results}

Fish mortality (16.7\%) due to parasitic infection began $3 \mathrm{~d}$ after temperature reached $24{ }^{\circ} \mathrm{C}$. Mortality exceeded $50 \%$ a day later, and by the fifth day, mortality was more than $80 \%$ (Fig. 1a). The analyzed yellowtails showed $100 \%$ parasitic prevalence with mean intensity of $766 \pm$ 500 parasites per fish throughout the experiment (Fig. 1b). Histological analyses indicated attachment of A. ocellatum to the gill, and gill tissue reactions were characterized by inflammation of vascular structures, epithelial detachment, proliferation of mucous cells (Fig. 2a), hyperplasia, fusion of secondary lamellae (Fig. 2b, c), and telangiectasia (Fig. 2d).

Blood parameters of infected fish revealed $100 \%$ parasitic prevalence, with mean intensity of $882.2 \pm 265.1$ parasites per fish. Counts for RBC, hemoglobin concentration, $\mathrm{MCHC}$, glucose concentration, and triglycerides were significantly higher $(P<0.05)$ in infected fish relative to healthy fish. In addition, mean corpuscular volume, total protein, albumin, and globulin were significantly lower $(P<0.05)$ in infected fish than in healthy fish. No significant differences were found in HCT, MCH, and WBC counts $(P>0.05)$ between healthy and infected fish. However, in the WBC counts an increase of $1.52 \times 10^{3} \mathrm{~mm}^{3}$ was observed in the infected fish group (Table 1).

\section{Análisis de los parámetros sanguíneos}

El HCT se midió con una muestra de sangre en un tubo capilar heparinizado llenado a 2/3 (LEEX Equipment, México). El tubo fue sellado y colocado por $10 \mathrm{~min}$ en una centrífuga de microhematocrito a 7,000 rpm (Premiere XC-3012, México). El paquete celular fue medido con un lector para hematocrito y reportado como porcentaje (Del Rio-Zaragoza et al. 2008). La hemoglobina en los eritrocitos se determinó usando un analizador HemoCue $\mathrm{Hb} 201 \mathrm{de}$ acuerdo con las instrucciones del fabricante (HemoCue AB; Angelholm, Suecia). Se utilizó el método de Natt y Herrick (1952) para el recuento total de GR y de GB más trombocitos. Las células fueron contadas con un hemocitómetro Neubauer bajo microscopio óptico (Zeiss, PrimoStar; Göttingen, Alemania). El volumen corpuscular medio, la hemoglobina corpuscular media (HCM) y la concentración de hemoglobina corpuscular media (CHCM) fueron calculados a partir de fórmulas estándar con los datos de HCT, GR y hemoglobina. Las proteínas totales, la albúmina, la globulina, la glucosa y los triglicéridos del suero sanguíneo fueron determinados usando los ensayos de un kit colorimétrico (MexLab Group; Jalisco, México) y siguiendo las instrucciones del fabricante. La globulina se obtuvo restando el valor de albúmina del total de proteínas.

\section{Análisis estadístico}

Todas las mediciones fueron realizadas en triplicado para cada pez. La prueba de normalidad y la prueba- $t(P<0.05)$ fueron aplicadas a los datos usando el software SigmaStat 4 (Systat Software; San Jose, CA, EUA). Todos los datos porcentuales fueron transformados a arcoseno antes de las comparaciones estadísticas. Para aquellos datos que no mostraron una distribución normal, se usó una prueba no paramétrica (prueba de suma de rangos de Mann-Whitney).

\section{Resultados}

La mortalidad de peces (16.7\%) debido a los parásitos inició $3 \mathrm{~d}$ después de que la temperatura alcanzó $24{ }^{\circ} \mathrm{C}$. La mortalidad superó el $50 \%$ un día después, y al quinto día, la mortalidad fue de más del $80 \%$ (Fig. 1a). Los juveniles de jurel analizados presentaron una prevalencia parasitaria del $100 \%$ con una intensidad media de $766 \pm 500$ parásitos por pez durante el experimento (Fig. 1b). El análisis histológico indicó la adherencia de A. ocellatum a la branquia, y las reacciones del tejido branquial se caracterizaron por la inflamación de estructuras vasculares, el desprendimiento epitelial, la proliferación de células mucosas (Fig. 2a), la hiperplasia, la fusión de lamelas secundarias (Fig. 2b, c) y la telangiectasia (Fig. 2d).

Los parámetros sanguíneos de los peces parasitados revelaron un $100 \%$ de prevalencia con una intensidad media de $882.2 \pm 265.1$ parásitos por pez. Los recuentos de GR, la 


\section{Discussion}

Mexico has great potential for commercial mariculture operations and land-based hatchery production. However, mariculture operations are prone to parasitic infestations that can impair fish health, generally causing mortality. In this study we assessed the effect of a parasitic outbreak of A. ocellatum that resulted in mortality, induced gill damage, and changes in blood of juvenile yellowtail reared in experimental conditions. The A. ocellatum outbreaks found in our study have also been frequently observed in the bullseye puffer (Sphoeroides annulatus), spotted rose snapper (Lutjanus guttatus), Pacific red snapper (Lutjanus peru), yellow snapper (Lutjanus argentiventris), and mullet snapper (Lutjanus aratus), with nearly $100 \%$ mortality rate (Pérez-Urbiola et al. 2008, Fajer-Ávila et al. 2011). Rearing conditions beyond the optimum range for juvenile $S$. dorsalis, such as high temperatures $\left(>24{ }^{\circ} \mathrm{C}\right)$ and poor water quality, are likely to cause stress and are thus thought to promote protozoan outbreaks. In this context, our results highlighted a cumulative mortality rate of $100 \% 8 \mathrm{~d}$ postinfection, where highest mortality occurred
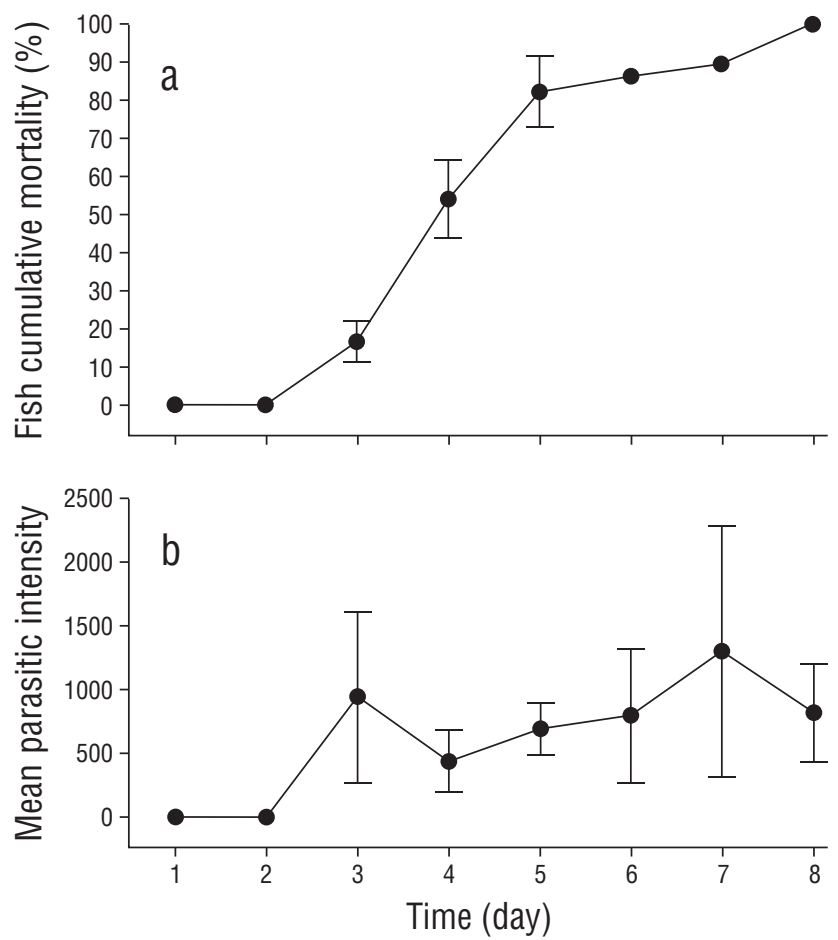

Figure 1. (a) Percent cumulative mortality of juvenile yellowtail Seriola dorsalis during $8 \mathrm{~d}$ of exposure to Amyloodinium ocellatum and (b) mean parasitic intensity of $A$. ocellatum on right side S. dorsalis gills.

Figura 1. (a) Porcentaje acumulado de la mortalidad de juveniles de jurel Seriola dorsalis durante $8 \mathrm{~d}$ de exposición a Amyloodinium ocellatum y (b) intensidad parasitaria media de A. ocellatum en las branquias izquierdas de $S$. dorsalis. concentración de hemoglobina, la CHCM, concentración de glucosa y triglicéridos fueron significativamente más elevados $(P<0.05)$ en peces parasitados en relación a los peces sanos. Además, el volumen corpuscular medio, las proteínas totales, la albúmina y la globulina fueron significativamente más bajos $(P<0.05)$ en los peces parasitados que en los peces sanos. No se encontraron diferencias significativas en los recuentos de HCT, HCM y GB $(P>0.05)$ entre peces sanos y parasitados. Sin embargo, en el recuento de GB se observó un incremento de $1.52 \times 10^{3} \mathrm{~mm}^{3}$ en el grupo de peces parasitados (Tabla 1).

\section{DisCUSIÓn}

México tiene gran potencial para las operaciones de maricultura comercial, así como para la producción en criadero. Sin embargo, las operaciones de maricultura son vulnerables a incidencias parasitarias que pueden perjudicar la salud de los peces y, generalmente, causar mortalidades. En este estudio evaluamos el efecto de un brote parasitario de A. ocellatum que provocó la mortalidad, la inducción de daño en las branquias y cambios en la sangre de juveniles de jurel criados bajo condiciones experimentales. Los brotes de $A$. ocellatum que se observaron en nuestro estudio han sido frecuentemente observados en el botete diana (Sphoeroides annulatus), el pargo lunarejo (Lutjanus guttatus), el pargo rojo del Pacífico (Lutjanus peru), el pargo amarillo (Lutjanus argentiventris) y el pargo raicero (Lutjanus aratus), con una tasa de mortalidad de casi el 100\% (Pérez-Urbiola et al. 2008, Fajer-Ávila et al. 2011). Las condiciones de crianza fuera del intervalo óptimo para los juveniles de $S$. dorsalis, como las altas temperaturas $\left(>24^{\circ} \mathrm{C}\right)$ y la mala calidad del agua, probablemente causan estrés, por lo cual son consideradas como elementos que promueven los brotes de protozoos. En este contexto, nuestros resultados resaltaron una tasa de mortalidad acumulada del $100 \%$ a los $8 \mathrm{~d}$ de la infestación, y la más alta mortalidad se observó entre el cuarto y quinto día después de la detección del parásito. Aunque la intensidad media de $A$. ocellatum fue más baja que la intensidad observada en el tercer día, las diferencias no fueron estadísticamente significativas. De modo similar, en los juveniles del mero leopardo (Mycteroperca rosacea) y del tambor rojo (Sciaenops ocellatum) la proliferación de este dinoflagelado resultó en una tasa de mortalidad acumulada del $89.5 \%$ y $90.0 \%$, respectivamente, $7 \mathrm{~d}$ después de la infección, y la mortalidad más alta se observó a las $48 \mathrm{~h}$ en ambas especies ( $\mathrm{Li}$ et al. 2005, Reyes-Becerril et al. 2008). En el besugo también se observó una alta tasa de mortalidad después de $2 \mathrm{~d}$ de la detección de Amyloodinium (Soares et al. 2012).

La infección de A. ocellatum en los jureles se caracterizó por signos iniciales de falta de apetito, seguidos por opacidad de la piel con manchas blanquizcas. Los peces empezaron a nadar de lado frotándose contra las paredes del tanque. Se observó también un movimiento rápido de las branquias. 

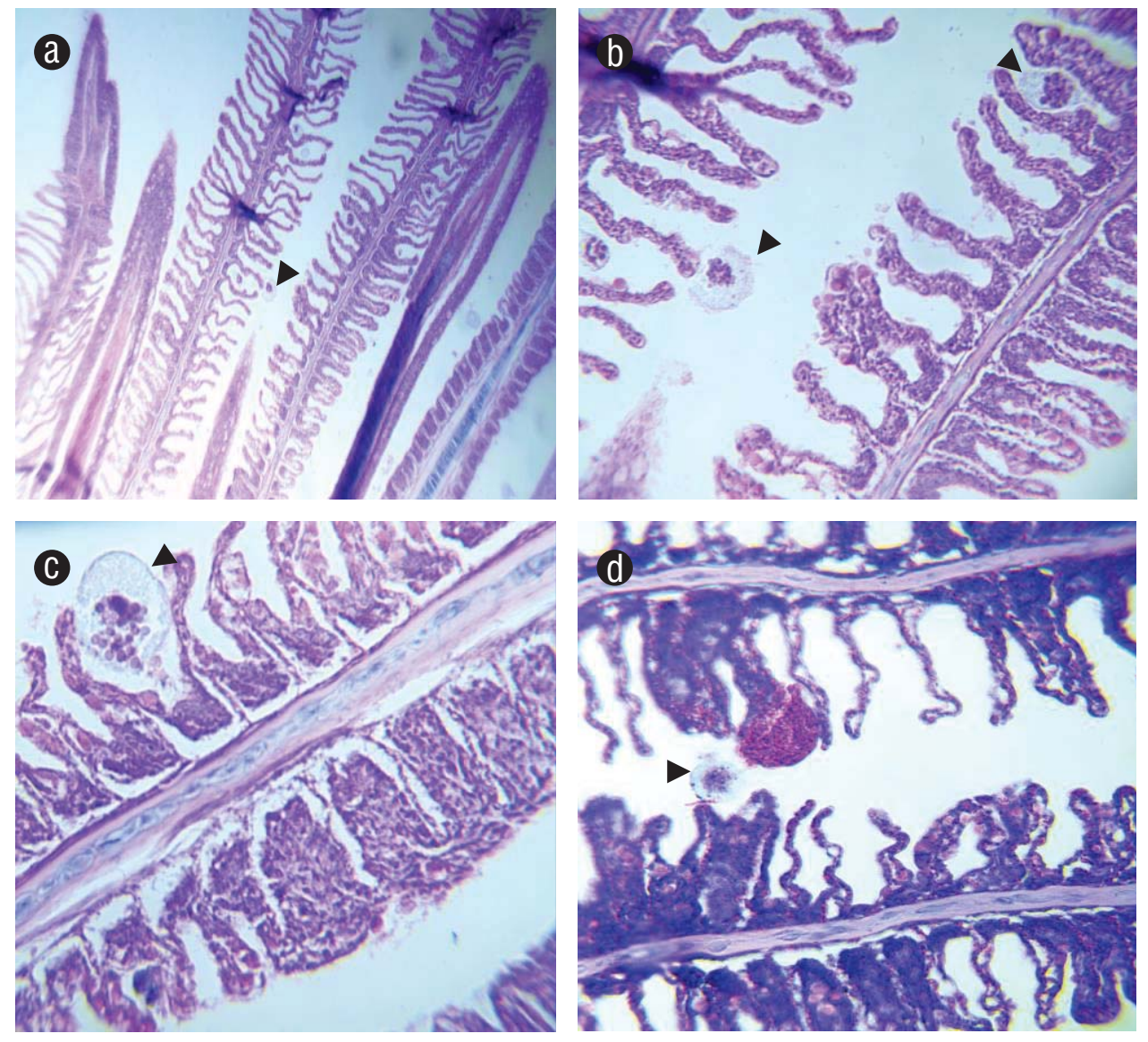

Figure 2. Histopathology of the second gill arch of juvenile yellowtail Seriola dorsalis associated with Amyloodinium ocellatum. (a) Gill lamellae with inflammation of vascular structures, proliferation of mucous cells, and hyperplasia in the distal parts of gill filaments; (b) gill lamellae with proliferative epithelial cells causing lamellar fusion; (c) gill filament with severe acute fusion of secondary gill lamellae; (d) gill filaments with fusion of secondary gill lamellae and telangiectasia. Arrow heads indicate the presence of A. ocellatum attached to the gill of the host. Haematoxylin and eosin stain. Images at $100 \times(\mathbf{a})$ and $400 \times(\mathbf{b}, \mathbf{c}, \mathbf{d})$.

Figura 2. Histopatología del segundo arco branquial de juveniles de jurel Seriola dorsalis asociados a Amyloodinium ocellatum. (a) Lamelas branquiales con las estructuras vasculares inflamadas, proliferación de las células mucosas e hiperplasia en las partes distales de los filamentos branquiales; (b) lamelas branquiales con células epiteliales proliferativas ocasionando fusión lamelar; (c) filamento branquial con una fusión aguda severa de las lamelas branquiales secundarias; (d) lamelas branquiales con la fusión de las lamelas branquiales secundarias y telangiectasia. Las flechas indican la presencia de Amyloodinium ocellatum anclado a la branquia del huésped. Tinción con hematoxilina y eosina. Imágenes a $100 \times($ a) y $400 \times(\mathbf{b}, \mathbf{c}, \mathbf{d})$.

between the fourth and the fifth day after detection of the parasite. Though mean intensity of A. ocellatum was lower than intensity on the third day, differences were not statistically significant. Similarly, in juvenile leopard groupers (Mycteroperca rosacea) and the juvenile red drum (Sciaenops ocellatum), proliferation of this dinoflagellate resulted in a cumulative mortality rate of $89.5 \%$ and $90.0 \%$, respectively, $7 \mathrm{~d}$ post-infection, where highest mortality occurred after $48 \mathrm{~h}$ in both species ( $\mathrm{Li}$ et al. 2005, Reyes-Becerril et al. 2008). A high mortality rate was also observed in seabream $2 \mathrm{~d}$ after Amyloodinium was detected (Soares et al. 2012).

Amyloodinium ocellatum infection in yellowtails was characterized by initial signs of appetite loss, followed by skin opacity with whitish spots. The fish started swimming
El comportamiento de S. dorsalis severamente parasitados durante el presente estudio fue similar al reportado por Kuperman y Matey (1999) en que el pez luchaba por captar oxígeno rápidamente, nadaba espásticamente y constantemente en la superficie antes de hundirse de nuevo al fondo, saltaba fuera del agua y finalmente perdía el equilibrio y moría. Casos similares se han reportado para el pargo lunarejo (Ontiveros-García 2008).

Nuestros hallazgos revelaron una severa infección en juveniles de $S$. dorsalis, con cientos de parásitos por pez, y un $100 \%$ de prevalencia parasitaria en las branquias de los peces examinados. La intensidad de la infestación por A. ocellatum en los filamentos branquiales fue alta y similar a los números reportados por Kuperman y Matey (1999) y Reyes-Becerril et al. (2008). 
sideways and rubbing themselves against the tank walls. Fast gill movement was also observed. Behavior of severely infected S. dorsalis during the present study was similar to that reported by Kuperman and Matey (1999), where fish rapidly gasped for air, swam spastically and constantly at the surface before sinking back to the bottom, jumped out of the water, and finally lost equilibrium and died. Similar cases have also been observed for the spotted rose snapper (Ontiveros-García 2008).

Our findings revealed severe infection in juvenile S. dorsalis, with hundreds of parasites per fish and $100 \%$ parasite prevalence in the gills of examined fish. The intensity of infection by A. ocellatum on gill filaments was high and similar to the numbers reported by Kuperman and Matey (1999) and Reyes-Becerril et al. (2008).

Amyloodinium ocellatum infect gill and skin epithelia. Rhizoids, which are root-like structures that parasites use to penetrate and grasp and that probably do not absorb nutrients, anchor the parasite to the host cells. The stomopode could be a source of digestive enzymes that are injected into host cells or could serve as a feeding tentacle that gathers cell fragments severed by the pulling motion of the rhizoids (Noga and Levy 2006, Soares et al. 2012). High infection levels were further associated to severe inflammation, epithelial detachment, hyperplasia, fusion of secondary lamellae, telangiectasia, and proliferation of mucus cells in the gills of $S$. dorsalis. Similar responses, except telangiectasia, have also been observed for L. guttatus (Ontiveros-García 2008). Meagre (Argyrosomus regius) and seabream (Sparus aurata) gills showed extensive areas with hyperplasia and necrosis of the epithelium (Soares et al. 2012). Del Rio-Zaragoza et al. (2010) observed that gill parasite infection by dactylogyrid monogeneans could stimulate mucus production in the host as a protective mechanism in response to parasitism, as observed for $S$. dorsalis with A. ocellatum in our study. However, production of excess mucus could cause hypoxia, inducing gill dysfunction in fish and, consequently, the death of the fish.

In the present study, a notorious physiological response was the increment in several blood parameters in the infected group, such as the number of erythrocytes, hemoglobin, and $\mathrm{MCHC}$, likely associated with gas transport and absorption issues or hemoconcentration caused by gill damage, dehydration, or stress. Lower levels in these blood parameters indicate anemia or hemodilution due to osmoregulation impairment (Wedemeyer and McLeay 1981). These results are in agreement with previous reports on L. guttatus and M. rosacea (Ontiveros-García 2008, Reyes-Becerril et al. 2008). Mean corpuscular volume resulted in a low count in infected fish, which reflects the size of the red blood cells, expressed as the volume of erythrocytes. In the present study, cell size adjustment was observed, which indicated that as the number of parasites
Table 1. Blood parameters for healthy and infected (by Amyloodinium ocellatum) yellowtail Seriola dorsalis. Data are mean \pm standard deviation.

Tabla 1. Parámetros sanguíneos de jurel Seriola dorsalis sano y parasitado (Amyloodinium ocellatum). Los datos muestran la media \pm desviación estándar.

\begin{tabular}{lcc}
\hline Parameters $^{\mathrm{a}}$ & $\begin{array}{c}\text { Healthy fish } \\
(n=23)\end{array}$ & $\begin{array}{c}\text { Infected fish } \\
(n=12)\end{array}$ \\
\hline Hematocrit $(\%)$ & $51.91 \pm 5.70$ & $49.50 \pm 6.84$ \\
RBC $\left(\times 10^{6} \mathrm{~mm}^{3}\right)$ & $4.19 \pm 0.79$ & $5.22 \pm 1.13^{*}$ \\
Hemoglobin $\left(\mathrm{g} \cdot \mathrm{dL}^{-1}\right)$ & $13.69 \pm 2.01$ & $16.02 \pm 1.86^{*}$ \\
MCV $(\mathrm{fL})$ & $127.53 \pm 25.70$ & $99.21 \pm 28.20^{*}$ \\
MCH $(\mathrm{pg})$ & $33.63 \pm 7.69$ & $32.11 \pm 8.62$ \\
MCHC $\left(\mathrm{g} \cdot \mathrm{dL} \mathrm{L}^{-1}\right)$ & $26.75 \pm 5.16$ & $32.98 \pm 6.09^{*}$ \\
WBC $\left(\times 10^{3} \mathrm{~mm}^{3}\right)$ & $26.26 \pm 9.74$ & $27.78 \pm 6.03$ \\
Total protein $\left(\mathrm{g} \cdot \mathrm{dL} \mathrm{L}^{-1}\right)$ & $4.42 \pm 0.89$ & $3.19 \pm 0.42^{*}$ \\
Albumin $\left(\mathrm{g} \cdot \mathrm{dL}^{-1}\right)$ & $1.36 \pm 0.34$ & $1.13 \pm 0.10^{*}$ \\
Globulin $\left(\mathrm{g} \cdot \mathrm{dL}^{-1}\right)$ & $3.06 \pm 0.75$ & $2.06 \pm 0.38^{*}$ \\
Glucose $\left(\mathrm{mg} \cdot \mathrm{dL}^{-1}\right)$ & $64.25 \pm 12.01$ & $115.66 \pm 14.52^{*}$ \\
Triglycerides $\left(\mathrm{mg}^{2} \cdot \mathrm{dL} \mathrm{L}^{-1}\right)$ & $48.61 \pm 13.18$ & $141.36 \pm 45.43^{*}$ \\
\hline
\end{tabular}

${ }^{a} \mathrm{RBC}$, red blood cells; $\mathrm{MCV}$, mean corpuscular volume; $\mathrm{MCH}$, mean corpuscular hemoglobin; $\mathrm{MCHC}$, mean corpuscular hemoglobin concentration; WBC, white blood cells.

*Significant differences $(P<0.05) ; n=$ number of fish analyzed.

Amyloodinium ocellatum infecta la branquia y el epitelio de la piel. Los rizoides, que son estructuras parecidas a raíces que usan los parásitos para penetrar y anclarse pero que probablemente no absorben nutrientes, anclan al parásito a las células huéspedes. El estomopodio puede ser una fuente de enzimas digestivas que son inyectadas a las células huéspedes o puede servir como un tentáculo de alimentación que reúne fragmentos de células desprendidas por el movimiento de tracción de los rizoides (Noga y Levy 2006, Soares et al. 2012). Los altos niveles de infestación también fueron asociados a la inflamación severa, el desprendimiento epitelial, la hiperplasia, la fusión de las lamelas secundarias, la telangiectasia y la proliferación de células mucosas en las branquias de $S$. dorsalis. También se han observado respuestas similares, a excepción de la telangiectasia, en L. guttatus (Ontiveros-García 2008). Las branquias de la perca regia Argyrosomus regius y el besugo (Sparus aurata) mostraron extensas áreas con hiperplasia y necrosis del epitelio (Soares et al. 2012). Del Rio-Zaragoza et al. (2010) observaron que una infestación parasitaria en la branquia por los monogeneos de la familia Dactylogyridae podía estimular la producción de mucus en el huésped como un mecanismo de protección al parasitismo, tal como se observó en nuestro estudio para S. dorsalis con A. ocellatum. Sin embargo, la producción 
increased, the number of erythrocytes increased. However, the diameter of erythrocytes decreased, causing a compensatory response from erythrocytes. This response was possibly caused by higher oxygen demand from infected organisms, due to the likely deficient gas exchange as a consequence of the obstruction and epithelial damage by $A$. ocellatum trophozoites in S. dorsalis gill filaments (Ontiveros-García 2008). Glucose and triglyceride concentrations were significantly higher in infected fish than in healthy ones. In the case of glucose, hyperglycaemia in yellowtail likely lead to chronic stress. The effect of chronic stress by exposure to a continuous sublethal agent is a longterm effect that alters the immune response of the organism (Wendelaar-Bonga and Balm 1999). No differences were found in $\mathrm{HCT}, \mathrm{MCH}$, and $\mathrm{WBC}$ counts $(P>0.05)$ for juvenile $S$. dorsalis. However, in the WBC counts an increase of $1.52 \times 10^{3} \mathrm{~mm}^{3}$ was observed in the infected fish group. Similarly, a positive correlation between the level of infection by dactylogyrid monogeneans and the total number of leucocytes was also observed by Del Rio-Zaragoza et al. (2011), indicating that this is a protective response to parasitic stress. In addition, total protein, albumin, and globulin levels were significantly lower $(P<0.05)$ in infected fish than in healthy ones. The low total protein levels found in infected fish could be associated with the presence of A. ocellatum, and total protein could be used to evaluate the physiological state and general condition of the fish as previously suggested (De Pedro et al. 2005). The decrease in protein concentration has been attributed to illness (e.g., liver damage), decreased nutrient absorption, nutritional deficiency, starvation, and infectious diseases (Wedemeyer and McLeay 1981, Del Rio-Zaragoza et al. 2011).

Changes in blood parameters could offer a direct or indirect assessment of the health and physiological state of the organism. For this reason, evaluation of haematological and blood chemistry parameters could help detect diseases and sublethal conditions affecting fish under culture conditions (Del Rio-Zaragoza et al. 2010). The present findings demonstrated that $A$. ocellatum infection caused severe gill damage and thus affected gas exchange efficiency, resulting in blood parameters changes and, consequently, high mortality rates in a short-term period. This study is the first contribution to acknowledge the effect of A. ocellatum on juvenile yellowtail and provide valuable insights for future studies implementing strategies to prevent and control amyloodiniosis in this species, which is of high commercial value.

\section{ACKnowledgments}

This project was financed by PRODEP UABC-PTC-560. Roberto Flores-Aguilar, director of Ocean Baja Labs, donated the fish. Thanks are due to Dr. Alejandro Cabello-Pasini for reviewing the manuscript. de mucus en exceso podría causar hipoxia e inducir el mal funcionamiento de las branquias $\mathrm{y}$, consecuentemente, la muerte de los peces.

En el presente estudio, una respuesta fisiológica destacable fue el incremento de varios parámetros sanguíneos en el grupo infectado, como el número de eritrocitos, hemoglobina y CHCM, probablemente asociados a problemas de transporte y absorción de gases o hemoconcentración causada por daño de las branquias, deshidratación o estrés. Los bajos niveles de estos parámetros sanguíneos indican anemia o hemodilución debido al deterioro de la osmorregulación (Wedemeyer y McLeay 1981). Estos resultados concuerdan con informes anteriores sobre L. guttatus y $M$. rosacea (Ontiveros-García 2008, Reyes-Becerril et al. 2008). El volumen corpuscular medio dio lugar a un valor bajo en peces parasitados, lo cual refleja el tamaño de los glóbulos rojos, expresado como volumen de los eritrocitos. En el presente trabajo se observó un ajuste en el tamaño celular, el cual indicó que a medida que aumentó el número de parásitos también aumentó el número de eritrocitos. Sin embargo, el diámetro de los eritrocitos disminuyó, lo cual ocasionó una respuesta compensatoria de los eritrocitos. Esta respuesta fue posiblemente causada por la alta demanda de oxígeno de los organismos parasitados, debido a la probable deficiencia en el intercambio gaseoso como consecuencia de la obstrucción y el daño epitelial de los trofozoitos de A. ocellatum en los filamentos branquiales de $S$. dorsalis (Ontiveros-García 2008). La glucosa y la concentración de triglicéridos fue significativamente más elevada en los peces infectados que en los sanos. En el caso de la glucosa, la hiperglicemia en el jurel probablemente llevó al estrés crónico. El efecto del estrés crónico por exposición continua a un agente subletal es un efecto a largo plazo que altera la respuesta inmune del organismo (Wendelaar-Bonga y Balm 1999). No se encontraron diferencias entre los recuentos de HCT, HCM y GB $(P>0.05)$ para los juveniles de S. dorsalis. Sin embargo, se observó un incremento de $1.52 \times 10^{3} \mathrm{~mm}^{3}$ en el recuento de GB para el grupo de peces parasitados. De forma similar, Del Rio-Zaragoza et al. (2011) observaron una correlación positiva entre el nivel de infestación de los monogeneos de la familia Dactylogyridae y el número total de leucocitos, lo cual indica que ésta es una respuesta de protección al estrés parasitario. Además, los niveles de proteínas totales, albúmina y globulina fueron significativamente más bajos $(P<0.05)$ en peces parasitados que en los peces sanos. Los bajos niveles de proteínas totales encontrados en peces parasitados podrían estar asociados a la presencia de A. ocellatum, y las proteínas totales podrían usarse en la evaluación del estado fisiológico y la condición general de los peces, como se sugirió previamente (De Pedro et al. 2005). La disminución en la concentración de proteínas ha sido atribuida a una enfermedad (e.g., daño del hígado), la disminución en la absorción de nutrientes, la deficiencia nutricional, inanición y enfermedades infecciosas (Wedemeyer y McLeay 1981, Del Rio-Zaragoza et al. 2011). 


\section{REFERENCES}

Aiello P, D’Alba A. 1986. Amyloodinium ocellatum infestation in yellowtail, Seriola dumerili, intensively reared in sicily, Italy. Bull. Eur. Ass. Fish Pathol. 6(4): 110-111.

Bush AO, Lafferty KD, Lotz JM, Shostak AW. 1997. Parasitology meets ecology on its own terms: Margolis et al. revisited. J. Parasitol. 83(4): 575-583. https://doi.org/10.2307/3284227

De Pedro N, Guijarro AI, López-Patiño MA, Martínez-Álvarez R, Delgado MJ. 2005. Daily and seasonal variations in haematological and blood biochemical parameters in the tench Tinca tinca Linnaeus, 1758. Aquac. Res. 36(12): 11851196. https://doi.org/10.1111/j.1365-2109.2005.01338.x

Del Rio-Zaragoza OB, Fajer-Avila EJ, Almazán-Rueda P. 2010. Haematological and gill responses to an experimental infection of dactylogyrid monogeneans on the spotted rose snapper Lutjanus guttatus (Steindachner, 1869). Aquac. Res. 41(11): 1592-1601. https://doi.org/10.1111/j.1365-2109.2009.02471.x

Del Rio-Zaragoza OB, Fajer-Ávila EJ, Almazán-Rueda P, Abdo de la Parra MI. 2011. Hematological characteristics of the spotted rose snapper Lutjanus guttatus (Steindachner, 1869) healthy and naturally infected by dactylogyrid monogeneans. Tissue Cell 43(3): 137-142. https://doi.org/10.1016/j.tice.2011.01.002

Del Rio-Zaragoza OB, Hernández-Rodríguez M, Bückle-Ramírez LF. 2008. Thermal stress effect on tilapia Oreochromis mossambicus (Pisces: Cichlidae) blood parameters. Mar. Freshwat. Behav. Physiol. 41(2): 79-89. https://doi.org/10.1080/10236240801896223

Fajer-Ávila EJ, Abad-Rosales SM, Medina-Guerrero RM, Betancourt-Lozano M. 2011. Avances sobre las investigaciones parasitológicas en el cultivo de pargo flamenco y botete diana Sinaloa, México. In: Ruiz-Luna A, Berlanga-Robles CA, Betancourt-Lozano M (eds.), Avances en Acuicultura y Manejo Ambiental. Trillas, Mexico, pp. 71-92.

Fajer-Avila EJ, Del Rio-Zaragoza OB, Betancourt-Lozano M. 2012. Parasitic diseases in cultured marine fish in Northwest Mexico. In: Carvalho E (ed.), Health and Environment in Aquaculture. InTechOpen, Rijeka, Croatia, pp. 63-94. https://www.intechopen.com/books/health-and-environmentin-aquaculture/parasitic-diseases-in-cultured-marine-fish-innorthwest-mexico

Guerra-Olvera FM, Viana MT. 2015. Effect of dietary cholesterol content on growth and its accumulation in liver and muscle tissues of juvenile yellowtail kingfish (Seriola lalandi) $=$ Efecto del contenido de colesterol en las dietas y su acumulación en hígado y músculo en juveniles de jurel de Castilla (Seriola lalandi). Cienc. Mar. 41(2): 143-156.

http://dx.doi.org/10.7773/cm.v41i2.2514

Kuperman BI, Matey VE. 1999. Massive infestation by Amyloodinium ocellatum (Dinoflagellida) of fish in a highly saline lake, Salton Sea, California, USA. Dis. Aquat. Org. 39: $65-73$. http://dx.doi.org/10.3354/dao039065

Li P, Burr GS, Goff J, Whiteman KW, Davis KB, Vega RR, Neill WH, Gatlin DM III. 2005. A preliminary study on the effects of dietary supplementation of brewers yeast and nucleotides, singularly or in combination, on juvenile red drum (Sciaenops ocellatus). Aquac. Res. 36(11): 1120-1127.

http://dx.doi.org/10.1111/j.1365-2109.2005.01333.x
Los cambios en los parámetros sanguíneos podrían ofrecer una evaluación directa o indirecta de la salud y el estado fisiológico del organismo. Por ésta razón, la evaluación de parámetros hematológicos y químicos de la sangre podría ayudar a detectar enfermedades y condiciones subletales que afectan a los peces en condiciones de cultivo (Del Rio-Zaragoza et al. 2010). Los resultados del presente estudio demostraron que la infección por A. ocellatum causó serios daños en las branquias y, por tanto, afectó la eficiencia del intercambio gaseoso, lo cual resultó en cambios de los parámetros sanguíneos y, por lo tanto, en altas tasas de mortalidad en un período corto. Este estudio es la primera contribución en reconocer el efecto de A. ocellatum en juveniles de jurel y en proporcionar información valiosa para estudios futuros que ayudarán a implementar estrategias de prevención y control de la amyloodiniosis en esta especie de gran valor comercial.

\section{Agradecimientos}

Este proyecto fue financiado por PRODEP UABC-PTC-560. Roberto Flores-Aguilar, director de Ocean Baja Labs, donó los peces. Agradecemos al Dr. Alejandro Cabello-Pasini su revision al manuscrito.

Traducido al español por Monica Bricelj.

Lom J, Dykova I. 1992. Protozoan Parasites of Fish. Elsevier, Amsterdam, Netherlands. 315 pp.

Martinez-Takeshita N, Purcell CM, Chabot CL, Craig MT, Paterson CN, Hyde JR, Allen LG. 2015. A tale of three tails: Cryptic speciation in a globally distributed marine fish of the genus Seriola. Copeia 103(2): 357-368.

https://doi.org/10.1643/CI-124-224

Montero FE, Crespo S, Padrós F, De la Gándara F, García A, Raga JA. 2004. Effects of the gill parasite Zeuxapta seriolae (Monogenea: Heteraxinidae) on the amberjack Seriola dumerili Risso (Teleostei: Carangidae). Aquaculture 232(14): $153-163$.

https://doi.org/10.1016/S0044-8486(03)00536-2

Moran D, Pether SJ, Lee PS. 2009. Growth, feed conversion and faecal discharge of yellowtail kingfish (Seriola lalandi) fed three commercial diets. N. Z. J. Mar. Freshwat. Res. 43(4): 917-927. https://doi.org/10.1080/00288330909510050

Nakada MK. 2002. Yellowtail culture development and solutions for the future. Rev. Fish. Sci. 10(3-4): 559-575. https://doi.org/10.1080/20026491051794

Natt MP, Herrick CA. 1952. A new blood diluent for counting erythrocytes and leukocytes of the chicken. Poult. Sci. 31(4): 735-738. https://doi.org/10.3382/ps.0310735

Noga EJ, Levy MG. 2006. Phylum Dinoflagellata. In: Woo PTK (ed.), Fish Diseases and Disorders. 2nd ed. Protozoan and Metazoan Infections; vol. 1. CAB International, Wallingford, (Oxfordshire, United Kingdom), pp. 16-45. 
Ontiveros-García LA. (2008). Efecto de las infestaciones causadas por el protozoario Amyloodinium ocellatum (Brown, 1931) sobre los parámetros sanguíneos y filamentos branquiales del pargo lunarejo, Lutjanus guttatus (Steindachner, 1869). MSc thesis, Centro de Investigación en Alimentación y Desarrollo, Mazatlán (Sinaloa, Mexico), 101 pp.

Pérez-Urbiola JC, Inohuye-Rivera RB, Muhlia-Melo AF. 2008. Catálogo de parásitos de pargos Lutjanus spp. en Bahía de La Paz e Isla Cerralvo, Baja California Sur, México. Centro de Investigaciones Biológicas del Noroeste, Baja California Sur (Mexico).

Poortenaar CW, Hooker SH, Sharp N. 2001. Assessment of yellowtail kingfish (Seriola lalandi) reproductive physiology, as a basis for aquaculture development. Aquaculture 201(3-4): 271-286. https://oi.org/10.1016/S0044-8486(01)00549-X

Reyes-Becerril M, Ascencio-Valle F, Alamillo E, Hirono I, Kondo H, Jirapongpairoj W, Angulo C. 2015. Molecular cloning and comparative responses of Toll-like receptor 22 following ligands stimulation and parasitic infection in yellowtail (Seriola lalandi). Fish. Shellfish. Immun. 46(2): 323-333. https://doi.org/10.1016/j.fsi.2015.06.020

Reyes-Becerril M, Tovar-Ramírez D, Ascencio-Valle F, CiveraCerecedo R, Gracia-López V, Barbosa-Solomieu V. 2008. Effects of dietary live yeast Debaryomyces hansenii on the immune and antioxidant system in juvenile leopard grouper Mycteroperca rosacea exposed to stress. Aquaculture 280 (1-4): 39-44. https://doi.org/10.1016/j.aquaculture.2008.03.056
Rombenso AN, Trushenski JT, Jirsa D, Drawbridge M. 2016. Docosahexaenoic acid (DHA) and arachidonic acid (ARA) are essential to meet LC-PUFA requirements of juvenile California Yellowtail (Seriola dorsalis). Aquaculture 463: 123-134. https://doi.org/10.1016/j.aquaculture.2016.05.004

[SEPESCABC] Secretaría de Pesca y Acuacultura de Baja California. 2017. Reporte de Producción Pesquera y Acuícola de Baja California: Sistema SIPESCA/CONAPESCA 2016. SNIDRUS/OEIDRUS Pesca y Acuacultura, Baja California (Mexico).

Soares F, Quental-Ferreira H, Moreira M, Cunha E, Ribeiro L, Pousão-Ferreira P. 2012. First report of Amyloodinium ocellatum in farmed meagre (Argyrosomus regius) Bull. Eur. Ass. Fish Pathol. 32(1): 30-33.

Thoney DA, Hargis TWJ Jr. 1991. Monogenea (platyhelminthes) as hazards for fish in confinement. Annu. Rev. Fish Dis. 1: 133-153. https://doi.org/10.1016/0959-8030(91)90027-H

Wedemeyer GA, McLeay DJ. 1981. Methods for determining the tolerance of fishes to environmental stressors. In: Pickering AD (ed.), Stress and Fish. Academic Press, New York (NY), pp. 247-275.

Wendelaar-Bonga SE, Balm PHM. 1999. Histological and histopathological effects of stress. In: Balm PHM (ed.), Stress Physiology in Animals. CRC Press, Boca Raton (FL), pp.179-204.

Received February 2018, accepted October 2018. 